\title{
As relações de poder na atenção obstétrica e neonatal: perspectivas para o parto e o nascimento humanizados
}

The power relations in obstetric attention: perspectives for humanized labor and birth

\author{
Las relaciones de poder em la atención obstétrica: perspectivas para el parto y el \\ nascimiento humanizados
}

Greice Machado Pieszak*, Giovana Calcagno Gomes², Andressa Peripolli Rodrigues³, Laís Antunes Wilhelm ${ }^{4}$

\section{RESUMO}

Objetivo: refletir a respeito das relações de poder que permeiam a atenção obstétrica e neonatal, discutindo as perspectivas para o parto e o nascimento humanizados. Método: estudo teórico e reflexivo, ancorado em referenciais sobre o processo de parturição e sobre poder, especialmente, nos estudos de Foucault: A Microfísica do Poder e o sujeito e o poder. Resultados: destaca-se que as relações de poder que permeiam a assistência obstétrica se sustentam no modelo hegemônico e no predomínio de práticas intervencionistas, ancorada nas rotinas e padrões que necessitam de aporte científico e cuidado humanizado. Revela-se que o poder profissional em relação à parturição e o protagonismo feminino, define a genealogia dessa relação. Quanto às perspectivas de rompimento do paradigma dominante, tem-se a necessidade de reapropriação do corpo e do parto feminino, por meio de novas modalidades de assistência que potencializam o poder e o saber da mulher. Considerações finais: acredita-se que a assistência obstétrica baseada em evidências científicas e nas tecnologias educacionais oportunizem mudanças e rompimentos pragmáticos no processo de parto e nascimento.

Palavras-chave: Equipe de Assistência ao Paciente, Enfermagem, Enfermagem Obstétrica, Parto, Obstetrícia; Recém-nascido.

\begin{abstract}
Aim: to reflect on the power relations that permeate obstetric and neonatal care, discussing the perspectives for humanized childbirth and birth. Method: a theoretical and reflexive study, anchored in references about the process of parturition and power, especially in Foucault's studies: The Microphysics of Power and the subject and the power. Results: we highlight that the power relations that permeate obstetric care are based on the hegemonic model and the predominance of interventionist practices, anchored in the routines and patterns that require scientific input and humanized care. It is revealed that the professional power in relation to parturition and female protagonism, defines the genealogy of this relationship. As for the prospects of breaking the dominant paradigm, there is a need to reappropriate the female body and childbirth, through new forms of assistance that empower women's power and knowledge. Final considerations: it is believed that obstetrical care based on scientific evidence and educational technologies allows for pragmatic changes and ruptures in the process of childbirth and birth.
\end{abstract}

Keywords: Patient Care Team; Nursing; Obstetric Nursing; Parturition; Obstetrics; Newborn.

\footnotetext{
1 Universidade Regional Integrada do Alto Uruguai e das Missões- URI Campus de Santiago, Santiago, Rio Grande do Sul/ Brasil. *E-mail: greicepieszak@gmail.com

2 Universidade Federal do Rio Grande (FURG), Rio Grande, Rio Grande do Sul/ Brasil.

3 Instituto Federal de Educação, Ciência e Tecnologia Farroupilha - Santo Ângelo, Rio Grande do Sul/ Brasil.

4 Universidade Federal de Santa Maria (UFSM), Santa Maria, Rio Grande do Sul/Brasil.
} 


\section{RESUMEN}

Objetivo: reflexionar sobre las relaciones de poder que permean la atención obstétrica y neonatal, discutiendo las perspectivas para el parto y el nacimiento humanizados. Método: el estudio sobre el proceso de parturition and power, especialmente en los estudios de Foucault: The Microphysics of Power and the subject and the power. Resultados: se resalta que las relaciones de energía que impregnan el obstáculo obstetricio se basan en el modelo y el predominio de intervenciones prácticas, anchadas en las rutinas y los patrones que requieren un análisis científico y humanizado. Se muestra que la habilidad de trabajar en relación con la parturición y el protagonismo de género, establece la genealogía de esta relación. En el caso de que los prospectos de la evolución del paradigma dominante, hay que tener que volver a aplicar el cuerpo masculino y la maternidad, a través de nuevos formularios de ayuda que permita a las mujeres y el conocimiento. Consideraciones finales: se cree que la asistencia obstétrica basada en evidencias científicas y en las tecnologías educativas oportunicen cambios y rompimientos pragmáticos en el proceso de parto y nacimiento.

Palavras-clave: Grupo de Atención al Paciente; Enfermería; Enfermería Obstétrica; Parto; Obstetricia; Recién Nascido.

\section{INTRODUÇÃO}

No Brasil, $98 \%$ dos nascimentos acontecem em instituições hospitalares e a cada ano ocorrem três milhões de nascimentos, envolvendo uma parcela significativa da população brasileira em uma vivência singular que incluí parturientes, seus bebês, famílias e o seu meio social (BRASIL, 2017). Porém, no Brasil contemporâneo, os nascimentos não tem sido uma experiência natural às mulheres, uma vez que a hospitalização delas para o parto encontra-se permeada de intervenções e da medicalização do corpo feminino (RIESCO MLG, 2014). Além de ser caracterizada por um modelo assistencial centrado na institucionalização, não na mulher e no seu parto. A assistência obstétrica é assumida por um autoritarismo profissional, biomédico, tecnocrático e dominante, ancorada nas rotinas, padrões e despersonalização pela concentração do saber hegemônico (FLOYD RD, et al., 2010; GARRE JMH E PÉREZ PE, 2015).

Logo, a atenção ao parto e ao nascimento envolve a miríade corpo e parto, que abarca experiências que são coincidentes às questões culturais e as relações de gênero (AQUINO EML, 2014). Esse fato contribui para que diversas intervenções sejam realizadas ao longo desse processo, como é o caso das cirurgias cesarianas, que evidencia um uso abusivo de tecnologias que, muitas vezes, culminam em iatrogenias, gerando repercussões no protagonismo feminino e na experiência singular de parir (DODOU HD, et al., 2017).

Neste panorama da assistência obstétrica que padroniza a atenção ao parto com rotinas hospitalares. Há necessidade da transcendência, do resgate da autonomia da mulher, devolvendo o pertencimento do seu corpo, do seu parto e das suas necessidades. Para isso, é importante rever o processo de sujeição e as relações de saber/poder que se estabelecem no cenário de parturição e nascimento. É neste contexto de atendimento obstétrico e de relações de micropoderes entre os profissionais de saúde que se localiza o foco de interesse desta reflexão e questiona-se: como se revelam as relações de poder na atenção obstétrica e neonatal? Tem-se como objetivo do estudo refletir a respeito das relações de poder que permeiam a atenção obstétrica e neonatal, discutindo as perspectivas para o parto e o nascimento humanizados.

\section{DISCUSSÃO e RESULTADOS}

Trata-se de um estudo teórico e reflexivo ancorado em referenciais sobre o processo de parturização (BRASIL, 2017; FLOYD RD, et al., 2010) e sobre poder, especialmente, nas obras de Foucault: A Microfísica do Poder (FOUCAULT M, 2014) e O sujeito e o Poder (FOUCAULT M, 1995). Neste arcabouço teórico, visualiza-se as diferentes perspectivas entre as relações de poder no âmbito da atenção obstétrica e neonatal, por meio de uma contextualização reflexiva. No âmbito obstétrico, os programas, políticas e 
portarias criadas ainda não conseguiram romper o paradigma hegemônico estruturado historicamente no Brasil, que é regido por valores biomédicos e por uma estrutura autoritária dos profissionais da saúde (FLOYD RD, et al., 2010). Sob essa perspectiva, verifica-se as mudanças que ocorrem a partir da hospitalização das mulheres para o parto, pois os profissionais de saúde atuantes em um modelo institucionalizado assumem ações de poder profissional em relação à parturição e essas ações passam a interferir no protagonismo feminino.

Neste sentido, Foucault M (2014) diz que as instituições, como os hospitais, tornaram-se ambientes privilegiados para ampliar as medidas disciplinares de maneira normativa, formando corpos dóceis e dominados. Ao definir a ideia do poder, o corpo humano e neste caso o corpo da parturiente, torna-se alvo dele, e passa a ser desarticulado e recomposto. E assim, é adaptado para se tornar útil e vulnerável às intervenções. Dessa forma, é possível inferir que controlar os corpos está diretamente associado ao poder e dominação (FOUCAULT M, 2014).

As repercussões do modelo de atenção obstétrica atual provocam alterações no processo de parturição que se relacionam à forma de cuidar de mulheres, recém-nascidos e suas famílias, afastando-as de seus saberes, experiências e práticas do parto e nascimento (COLLAÇO VS, et al., 2016).

Essa realidade, tolhida pelo modelo intervencionista e tecnocrático de assistência, também é reflexo da diversidade cultural que contempla o processo dos nascimentos contemporâneos (FEYER ISS, et al., 2013).

Neste panorama, a pesquisa nascer no Brasil, realizada em maternidades públicas, privadas e mistas, com 23.894 mulheres, revelou dados preocupantes sobre os nascimentos no país, indicando que $98 \%$ destes ocorrem em instituições hospitalares e índices de cesarianas de 52\% (AQUINO EML, 2014; FUJITA JALM et al., 2015).

Diante do cenário mundial, o país lidera o ranking de procedimentos cirúrgicos como via de parto, necessitando-se adequar às recomendações da Organização Mundial da Saúde (OMS) (OMS, 2015) que estabelece a taxa de 10 a $15 \%$ desse procedimento. Portanto, mesmo que a cesariana consista em um recurso que pode diminuir a mortalidade e morbidade materna e perinatal, não existem comprovações de que realizá-la de forma indiscriminada pode trazer benefícios às mulheres e bebês (TORRES JA, et al., 2014). A obstetrícia ainda está frente a esse modelo assistencial medicalizado, mesmo este sendo questionado pela própria OMS (GARRE JMH E PÉREZ PE, 2015).

Com relação ao parto vaginal, tem-se como intervenções, como a episiotomia (incisão feita com tesoura ou bisturi para ampliar o canal vaginal e que necessita de sutura); a obrigação de parir na posição de litotomia (decúbito dorsal), a manobra de Kristeller (exercício de força sobre o abdômen da mulher pelos profissionais de saúde). Ainda, o enema (lavagem intestinal), a infusão endovenosa de ocitocina sintética (rotineira, já na internação da paciente, para acelerar as contrações), a amniotomia (rompimento da bolsa amniótica artificialmente pelo profissional), a dilatação cervical (na avaliação ginecológica são forçadas as bordas do colo uterino), dentre outras. Tais práticas rotineiras exemplificadas não estão apoiadas positivamente pelas evidências científicas (BRASIL, 2017), mas são utilizadas de maneira indiscriminada pelos profissionais da saúde. Remete-se a padronização constante de procedimentos e rotinas o que desencadeia a sujeição às rotinas impostas no ambiente de trabalho, modelando o sujeito (FOUCAULT M, 2014).

Em contrapartida, sabe-se que as práticas consideradas positivas no trabalho de parto e parto, por vezes, não são aderidas pelos profissionais atuantes em Centros Obstétricos, como a oferta de alimentação ou líquido, a liberdade de movimentos, massagens e banho de conforto (AQUINO EML, 2014).

Ainda, a não permissão do acompanhante de livre escolha da mulher durante o trabalho de parto e parto. Este impedimento, por vezes ultrapassa as normas institucionais e restringe-se ao poder de decisão dos profissionais, apesar de existir legislação que ampara as gestantes e garante esse direito a elas (BRASIL, 2005). Esse poder de tomada de decisão acerca de autorizações é detido a partir de quem tem o conhecimento, neste caso o profissional de saúde, visto como uma autoridade na instituição hospitalar e visualizado como o detentor do poder (FOUCAULT M, 1995; 2014). 
Ainda, reflete-se acerca das práticas intervencionistas realizadas aos recém-nascidos no processo de nascimento, que também tem sido padronizadas e, muitas delas, realizadas sem respaldo científico (TORRES JA, et al., 2014), como a aspiração sistemática naso e orofaringeana, a passagem de sonda nasogástrica e retal para descartar atresias, dentre outras, que não são recomendadas de forma sistemática nos recém-nascidos saudáveis (BRASIL, 2017).

$E$ as normativas que causam benefícios aos neonatos e que são comprovadas cientificamente, não são realizadas em muitos hospitais. Destaca-se o clampeamento do cordão umbilical, que deve ser feito entre um a cinco minutos ou de forma fisiológica quando cessar a pulsação do cordão logo após o parto. Tal prática tem inúmeros benefícios, como a redução do risco de anemia, melhora dos níveis de hematócrito e da concentração de ferritina (BRASIL, 2017).

Outra recomendação da OMS e do Ministério da Saúde brasileiro é o estímulo ao contato pele a pele entre mães e filhos imediatamente ou logo após o nascimento. Porém, esse contato é impedido, devido à necessidade da realização de procedimentos de rotina como, pesar, medir e dar banho no recém-nascido. É recomendado que seja minimizada a separação de mãe e filho, sendo consideradas as circunstâncias clínicas de ambos (BRASIL, 2017).

As evidências científicas que comprovam que o benefício do contato pele a pele entre mães e filhos ultrapassa a criação do vínculo, mas também há interferência duradoura em relação ao efetivo desenvolvimento cognitivo, nas habilidades sociais da criança (DALSANT A, et al., 2015) e nas taxas de eficácia da amamentação exclusiva (NIELA-VILÉN H, et al., 2017), e para a mãe a protege da depressão pós-parto (RECK C, et al., 2016). Também, um estudo internacional enfatiza a importância de tal prática mesmo em nascimentos oriundos de cesarianas, em que o contato pele a pele entre mãe e filho deve acontecer; e no caso de impossibilidade da mãe, devido ao ato cirúrgico, os pais devem assumir esse papel junto aos seus filhos. Infelizmente, os resultados desse estudo não refletem a realidade brasileira (BRASIL, 2017; OMS, 2015; NIELA-VILÉN H, et al., 2017), pois, na maioria das vezes, mãe e recém-nascido são separados após o parto devido as práticas e rotinas hospitalares (RECK C, et al., 2016).

É importante ressaltar também que tem ocorrido mudanças expressivas nos atendimentos aos partos e nascimentos no Brasil, que devem ser compartilhadas e refletidas. Destaca-se que os movimentos em prol da saúde da mulher vêm repetidamente reivindicando a reapropriação feminina de seu corpo durante o parto e nascimento, aliado ao processo de desmedicalização desse importante evento às mulheres (FLOYD RD, et al., 2010). O Ministério da Saúde intensificou suas ações na tentativa de discutir este modelo de assistência e garantir o acesso às práticas de saúde sustentadas em bases científicas e no protagonismo da mulher na gestação, parto e puerpério. Além disso, é necessário habilidade e experiência do profissional que presta o cuidado sejam associadas a meta de alcançar as expectativas e necessidades dessas mulheres e suas famílias (BRASIL, 2017).

No entanto, percebe-se que essa mudança de paradigma do modelo tecnocrático será viável quando o entendimento do parto for modificado, passando a ser percebido como algo natural e um evento humano. É a partir dessa percepção que as mulheres resgatarão sua autonomia para exercerem a cidadania e propiciarem o seu direito de escolha durante o parto e nascimento (DECLERCQ E, 2014).

Destaca-se que, dentre as ações em prol da assistência humanizada e integral no cuidado obstétrico e neonatal, tem-se o incentivo a formação de profissionais que atendam aos mesmos. Neste cenário, inserem-se as enfermeiras obstetras e obstetrizes, consideradas importantes no processo assistencial (BRASIL, 2016; 2017; MOUTA RJO e PROGIANTI JM, 2017), com o objetivo de transformar o modelo medicalizado no campo obstétrico.

Assim, visualiza-se a enfermagem enquanto promotora da humanização do cuidado e integralidade da assistência (MOUTA RJO e PROGIANTI JM, 2017; SANTOS FAPS, et al., 2016), por meio da utilização de tecnologias educacionais, a exemplo, no pré-natal, que tem motivado casais para o autocuidado e o cuidado com o bebê desenvolvendo elevado nível de conhecimento e confiança para tal prática. Por conseguinte, a comunicação e interação entre o profissional e os sujeitos, incentivam práticas saudáveis, desestimulando 
práticas inadequadas (BARBOSA EMG, et al., 2016), além de contribuírem com o julgamento clínico do enfermeiro e seleção de prioridades para a promoção do cuidado e da assistência qualificada.

Também, enfatiza-se que os ambientes onde ocorrem os nascimentos têm sofrido modificações, destaca-se as modalidades de assistência em ambientes não hospitalares, como o parto domiciliar planejado ou centros de nascimento ou casas de parto. Os mesmos têm assumido a promoção e o resgate das características naturais e fisiológicas do parto e nascimento. Este surge na contemporaneidade como uma possibilidade de reaproximação entre ciência e tradição, habilidade e intuição. Que valorizam o processo fisiológico, no sentido de deixar a natureza agir, de estimular o casal a assumir o protagonismo do processo de parir, com o mínimo de intervenção, tendo o apoio e o suporte de uma equipe qualificada, caso necessitem (COLLAÇO VS, et al., 2016; FEYER ISS, et al., 2013; COLLAÇO VS, et al., 2017).

Quanto às casas de parto, onde a premissa é o trabalho em equipe, em que as profissões interagem, criam estratégias de cuidados e se disciplinam e estabelecem relações de poder para a mudança de paradigma. Assim, a disciplina é um exemplo do positivo sob a análise foucaultiana, pois colabora naquilo que é capaz de produzir efeitos (FOUCAULT M, 2014).

Ressalta-se a importância dos grupos e da rede de apoio à parturiente e família, uma vez que oportunizam lutas em prol do protagonismo do parto e a possibilidade de mudança de paradigma, atentando para as transformações que as subjetividades das mulheres têm passado e que lhes são comuns: corpo, sexualidade, integridade e direitos (COLLAÇO VS, et al., 2017).

$O$ atendimento humanizado e baseado em evidências científicas na atenção ao parto e nascimento é um direito humano e, nesse sentido, faz-se necessário que todas às mulheres, homens, obstetrizes, enfermeiras, médicos, doulas e prestadores de atendimento à saúde visualizem o parir e nascer como uma questão de direitos humanos (FLOYD RD, et al., 2010), somente assim a realidade obstétrica e neonatal será transformada.

\section{CONSIDERAÇÕES FINAIS}

A reapropriação reflexiva acerca das relações de poder permeadas na atenção obstétrica revelam um paradigma dominante e hegemônico estruturado e sustentado na atenção obstétrica intervencionista. Sob a perspectiva foucaultiana, refletiu-se sobre a propagação do poder profissional na atenção ao parto e nascimento hospitalar. Desvelou-se a medicalização do corpo da mulher e impossibilidade do protagonismo feminino que define a genealogia do poder. Acredita-se que a assistência baseada em evidências científicas, a adoção de tecnologias educacionais mediadas pela enfermagem obstétrica e até mesmo a construção de saberes entre as mulheres, oportunizem rompimentos e reconstruções na atenção obstétrica e neonatal atual. Assim, almeja-se uma assistência integral, com uma mudança na postura profissional, que ainda é fortemente sustentada pelo modelo biomédico.

\section{REFERÊNCIAS}

1. AQUINO EML. Para reinventar o parto e o nascimento no Brasil: de volta ao futuro. Cadernos de Saúde Pública, 2014; 30(Sup): 8-10.

2. BARBOSA EMG, et al. Tecnologias educativas para promoção do (auto) cuidado de mulheres no pós-parto. Revista Brasileira de Enfermagem, 2016; 69(3): 582-90.

3. BRASIL. Lei $n^{\circ} 11.108$, de 07 de abril de 2005. Altera a Lei no 8.080 , de 19 de setembro de 1990, para garantir às parturientes $\mathrm{o}$ direito à presença de acompanhante durante o trabalho de parto, parto e pós-parto imediato, no âmbito do Sistema Único de Saúde (SUS). Diário Oficial da República Federativa do Brasil, Brasília (DF), 2005.

4. BRASIL. Lei no 0516 de 23 de junho de 2016. Normatiza a atenção e a responsabilidade do Enfermeiro Obstetra e Obstetriz na assistência à gestante, parturiente, puérpera e recém-nascido nos Serviços de Obstetrícia. Brasília (DF), 2016.

5. BRASIL. Ministério da Saúde. Secretaria de Ciência, Tecnologia e Insumos Estratégicos. Departamento de Gestão e Incorporação de Tecnologias em Saúde. Diretrizes nacionais de assistência ao parto normal: versão resumida. Brasília: Minsitério da Saúde, 2017; 51p. 
6. COLLAÇO VS, et al. Parir e nascer num novo tempo: o cuidado utilizado no puerpério pela Equipe Hanami. Revista Mineira de Enfermagem, 2016; 20: e949.

7. COLLAÇO VS, et al. O significado atribuído pelo casal ao parto domiciliar planejado, assistido pelas enfermeiras Obstétricas da Equipe Hanami. Texto Contexto Enfermagem, 2017; 26(2): e6030015.

8. DALSANT A, et al. Maternal bonding in childhood moderates autonomic responses to distress stimuli in adult males. Behavioural Brain Research, 2015; 292: 428-31.

9. DODOU HD, et al. O cuidado à mulher no contexto da maternidade: caminhos e desafios para a humanização. Cuidado é Fundamental Online, 2017; 9(1):222-30.

10. FEYER ISS, et al. Rituais de cuidado realizados pelas famílias na preparação para a vivência do parto domiciliar planejado. Revista Brasileira de Enfermagem, 2013; 66(6): 879-86.

11. FLOYD RD, et al. The International Mother Baby Childbirth Initiative: a human rights approach to optimal Maternity Care. Tempus Actas de Saúde Coletiva, 2010; 4(4): 93-103.

12. FOUCAULT M. Microfísica do poder. 28 ed. São Paulo: Paz \& Terra, 2014; 432p.

13. FOUCAULT M. O sujeito e o Poder. Rio de Janeiro: Forense, 1995; 231p.

14. FUJITA JALM, et al. O enfrentamento da violência obstétrica e suas repercussões na prática de enfermeiras obstetras. Revista de Enfermagem UFPE online, 2015; 9(12): 1360-69.

15. MOUTA RJO, PROGIANTI JM. O processo de criação da Associação Brasileira de Obstetrizes e Enfermeiros Obstetras. Texto Contexto Enfermagem, 2017; 26(1): e5210015.

16. NIELA VH, NANCY F, AXELIN A. Hospital routines promote parent-infant closeness and cause separation in the birthing unit in the first 2 hours after birth: A pilot study. Birth, 2017; 44: 167-72.

17. OMS. Organização Mundial da Saúde. Declaração sobre Taxas de Cesáreas. Genebra, 2015.

18. RECK C, et al. Perceived parenting stress in the course of postpartum depression: the buffering effect of maternal bonding. Archives of Women Mental Health, 2016; 19: 473-82.

19. RIESGO MLG. Nascer no Brasil "em tempo": uma questão de hierarquia das intervenções no parto? Cadernos de Saúde Pública, 2014; 30(Sup): 17-47.

20. SANTOS FAPS, et al. Integralidade e atenção obstétrica no Sistema Único de Saúde (SUS): reflexão à luz da teoria da complexidade de Edgar Morin. Escola Anna Nery, 2016; 20(4): e20160094.

21. TORRES JA, et al. Caesarean section and neonatal outcomes in private hospitals in Brazil: comparative study of two different perinatal models of care. Cadernos de Saúde Pública, 2014; 30(Sup): 220-31. 